\title{
Rigidity Theorems of Clifford Torus
}

\author{
LUIZ A. M. SOUSA JR.
}

Instituto de Matemática, Universidade Federal do Rio de Janeiro

C.P. 68530 - 21945-970 Rio de Janeiro, RJ, Brazil

\begin{abstract}
Manuscript received on May 31, 2001; accepted for publication on June 8, 2001;
presented by MANFREDO DO CARMO
\end{abstract}

\begin{abstract}
Let $M$ be an n-dimensional closed minimally immersed hypersurface in the unit sphere $S^{n+1}$. Assume in addition that $M$ has constant scalar curvature or constant Gauss-Kronecker curvature. In this note we announce that if $M$ has $(n-1)$ principal curvatures with the same sign everywhere, then $M$ is isometric to a Clifford Torus $S^{1}\left(\sqrt{\frac{1}{n}}\right) \times S^{n-1}\left(\sqrt{\frac{n-1}{n}}\right)$.
\end{abstract}

Key words: minimal hypersurfaces, scalar curvature, principal curvatures, Clifford Torus.

\section{INTRODUCTION}

Let $M$ be an n-dimensional hypersurface in a unit sphere $S^{n+1}$. We choose a local ortonormal frame field $\left\{e_{1}, \ldots, e_{n+1}\right\}$ in $S^{n+1}$, so that, restricted to $M, e_{1}, \ldots, e_{n}$ are tangent to $M$. Let $w_{1}, \ldots, w_{n+1}$ denote the dual co-frame field in $S^{n+1}$. Then, in $M$

$$
w_{n+1}=0 \text {. }
$$

It follows from Cartan's Lemma that

$$
w_{n+1, i}=\sum_{j} h_{i j} w_{j}, h_{i j}=h_{j i} .
$$

The second fundamental form $h$ and the mean curvature $H$ of $M$ are defined by

$$
h=\sum_{i, j} h_{i j} w_{i} w_{j} e_{n+1} \text { and } H=\sum_{i} h_{i i} .
$$

E-mail: amancio@impa.br 
We recall that $M$ is by definition a minimal hypersurface if the mean curvature of $M$ is identically zero. The connection form $w_{i j}$ is characterized by the structure equations

$$
\left\{\begin{array}{l}
d w_{i}+\sum_{j} w_{i j} \wedge w_{j}=0, \quad w_{i j}+w_{j i}=0 \\
d w_{i j}+\sum_{k} w_{i k} \wedge w_{k j}=\Omega_{i j} \\
\Omega_{i j}=\frac{1}{2} \sum_{k l} R_{i j k l} w_{k} \wedge w_{l}
\end{array}\right.
$$

where $\Omega_{i j}$ (resp. $R_{i j k l}$ ) denotes the curvature form (resp. the components of the curvature tensor) of $M$. The Gauss equation is given by

$$
R_{i j k l}=\left(\delta_{i k} \delta_{j l}-\delta_{i l} \delta_{j k}\right)+\left(h_{i k} h_{i l}-h_{i l} h_{j k}\right) .
$$

The covariant derivative $\nabla h$ of the second fundamental form $h$ of $M$ with components $h_{i j k}$ is given by

$$
\sum_{k} h_{i j k} w_{k}=d h_{i j}+\sum_{k} h_{j k} w_{i k}+\sum_{k} h_{i k} w_{j k} .
$$

Then the exterior derivative of (1.1) together with the structure equations yield the following Codazzi equation

$$
h_{i j k}=h_{i k j}=h_{j i k}
$$

Similarly, we have the covariant derivative $\nabla^{2} h$ of $\nabla h$ with components $h_{i j k l}$ as follows

$$
\sum_{l} h_{i j k l} w_{l}=d h_{i j k}+\sum_{l} h_{l j k} w_{i l}+\sum_{l} h_{i l k} w_{j l}+\sum_{l} h_{i j l} w_{k l},
$$

and it is easy to get the following Ricci formula

$$
h_{i j k l}-h_{i j l k}=\sum_{m} h_{i m} R_{m j k l}+\sum_{m} h_{m j} R_{m i k l} .
$$

From now on, we assume that $M$ is minimal. Denote by $S=\sum_{i, j} h_{i j}^{2}$ the square of length of $h$. The components of the Ricci curvature and the scalar curvature are given respectively by

$$
\begin{gathered}
R_{i j}=(n-1) \delta_{i j}-\sum_{k} h_{i k} h_{j k} . \\
R=n(n-1)-S .
\end{gathered}
$$

It follows from (1.8) that $S$ is constant if and only if $R$ is constant. For any fixed point $p$ in $M$, we can choose a local orthonormal frame field $e_{1}, \ldots, e_{n}$ such that

$$
h_{i j}=\lambda_{i} \delta_{i j}
$$


Let $S=\sum_{i, j} h_{i j}^{2}$. The following formulas can be obtained by a direct computation (Peng and Terng 1983).

$$
\begin{gathered}
\Delta h_{i j}=(n-S) h_{i j} . \\
\frac{1}{2} \Delta S=\sum_{i, j, k} h_{i j k}^{2}-S(S-n) .
\end{gathered}
$$

The Gauss-Kronecker curvature $K$ of $M$ is defined by

$$
K=\operatorname{det}\left(h_{i j}\right)
$$

In this note we give a sketch of the proof of the following results.

THEOREM 1.1. Let $M$ be a closed minimal hypersurface with constant scalar curvature in $S^{n+1}$. If $M$ has $(n-1)$ principal curvatures with the same signal everywhere, then $M$ is isometric to a Clifford Torus $S^{1}\left(\sqrt{\frac{1}{n}}\right) \times S^{n-1}\left(\sqrt{\frac{n-1}{n}}\right)$.

COROLlaRY 1.2. Let $M$ be a closed minimal hypersurface with constant scalar curvature in $S^{5}$. Assume in addition that $M$ has Gauss-Kronecker curvature $K$ negative everywhere. Then $M$ is isometric to a Clifford Torus $S^{1}\left(\frac{1}{2}\right) \times S^{3}\left(\frac{\sqrt{3}}{2}\right)$.

COROLLARY 1.3. Let $M$ be a closed minimal hypersurface with constant scalar curvature in $S^{5}$. Assume $S>4$. Then there exists a point $p$ on $M$ such that $K(p) \geq 0$.

THEOREM 1.4. Let M be a closed minimal hypersurface with constant Gauss-Kronecker curvature in $S^{n+1}$. If $M$ has $(n-1)$ principal curvatures with the same signal everywhere, then $M$ is isometric to a Clifford Torus $S^{1}\left(\sqrt{\frac{1}{n}}\right) \times S^{n-1}\left(\sqrt{\frac{n-1}{n}}\right)$.

\section{SKETCH OF THE PROOF OF THEOREM 1.1}

By changing the orientation for $M$ and renumbering $e_{1}, \ldots, e_{n}$ if necessary, we may assume

$$
\lambda_{n}<0<\lambda_{1} \leq \lambda_{2} \leq \ldots \leq \lambda_{n-1} .
$$

Notice that $K \neq 0$, hence the following function is well defined

$$
F=\log \left|\operatorname{det}\left(h_{i j}\right)\right| \text {. }
$$

We compute the Laplacian of $F$ obtaining

$$
F_{k}=\sum_{i, j} h^{i j} h_{i j k} \text { and } \Delta F=\sum_{k} F_{k k}=\sum_{i, j, k}\left(-\sum_{l, m} h^{i m} h^{l j} h_{l m k} h_{i j k}+h^{i j} h_{i j k k}\right) .
$$


Since $S$ is constant, from (1.10), (1.11) and using the symmetry of $h_{i j k}$ for indices, we get

$$
\begin{gathered}
S \Delta F=-\sum_{i}\left(\frac{S}{\lambda_{i}^{2}}+\frac{n}{3}\right) h_{i i i}^{2}-\sum_{i \neq j}\left(\frac{S}{\lambda_{i}^{2}}+\frac{2 S}{\lambda_{i} \lambda_{j}}+n\right) h_{i i j}^{2}-\sum_{i=1}^{n-1}\left(\frac{S}{\lambda_{i}^{2}}+\frac{2 S}{\lambda_{i} \lambda_{n}}+n\right) h_{i i n}^{2}- \\
\sum_{j=1}^{n-1}\left(\frac{S}{\lambda_{n}^{2}}+\frac{2 S}{\lambda_{j} \lambda_{n}}+n\right) h_{n n j}^{2}-\frac{1}{3} \sum_{i \neq j \neq k}\left[\left(\frac{\lambda_{i}+\lambda_{j}+\lambda_{k}}{\lambda_{i} \lambda_{j} \lambda_{k}}\right) S+n\right] h_{i j k}^{2}-\frac{2 n}{3} S(S-n) .
\end{gathered}
$$

We need the following algebraic Lemma.

Lemma 2.1. Let $\lambda_{i}$ and $\beta_{i j}, i, j=1, \ldots, n$, be real numbers such that $\lambda_{n}<0<\lambda_{1} \leq \ldots \leq \lambda_{n-1}$ and $\sum_{i} \lambda_{i}=\sum_{i} \beta_{i j}=\sum_{i} \lambda_{i} \beta_{i j}=0, j=1, \ldots, n$. Let us fix $j \in\{1, \ldots, n-1\}$ and set $g_{j} \in\{1, \ldots, n\}$ the greatest index such that $\left|\beta_{i j}\right|=\max \left\{\left|\beta_{g_{j}}\right|, i=1, \ldots, n\right\}$. Then $\beta_{n j}=0$ if and only if $g_{j}=n$.

By differentiating $\sum_{i} h_{i i}=0$ and $\sum_{i, k} h_{i k}^{2}=S=$ const., we obtain

$$
\sum_{i} h_{i i j}=0 \text { and } \sum_{i} \lambda_{i} h_{i i j}=0, \quad j=1, \ldots, n .
$$

Therefore, defining $\beta_{i j}=h_{i i j}$, then $\lambda_{i}$ and $\beta_{i j}$ satisfy the conditions in Lemma 2.1. Consider the following subsets of $\{1, \ldots, n-1\}=I_{n-1}$ :

$$
A=\left\{j \in I_{n-1} ; g_{j}=j\right\}, B=\left\{j \in I_{n-1} ; g_{j} \notin\{j, n\}\right\}, C=\left\{j \in I_{n-1} ; g_{j}=n\right\} .
$$

Possibly, some of these subsets may be empty.

After re-enumeration of $e_{1}, \ldots, e_{n-1}$ if necessary, we can assume that

$$
A=\emptyset \text { or } A=\{1, \ldots, r\}, B=\emptyset \text { or } B=\{r+1, \ldots, t\}, C=\emptyset \text { or } C=\{t+1, \ldots, n-1\},
$$

where we adopt the following convention: if $A=\emptyset$, then $r=0$, if $B=\emptyset$, then $t=r$ and if $C=\emptyset$, then $t=n-1$. For instance, if $B=\emptyset$, then $A=\{1, \ldots, r\}$ and $C=\{r+1, \ldots, n-1\}$.

According to Lemma 2.1, (2.3) can be rewritten as

$$
\begin{aligned}
S \Delta F=-\sum_{i=r+1}^{n}\left(\frac{S}{\lambda_{i}^{2}}=\frac{n}{3}\right) h_{i i i}^{2}-\sum_{\substack{i \neq j \\
i, j \leq n-1 \\
(i, j) \neq\left(g_{j}, j\right), j=r+1, \ldots, t}}\left(\frac{S}{\lambda_{i}^{2}}+\frac{2 S}{\lambda_{i} \lambda_{j}}+n\right) h_{i i j}^{2}- \\
\sum_{i=1}^{n-1}\left(\frac{S}{\lambda_{i}^{2}}+\frac{2 S}{\lambda_{i} \lambda_{n}}+n\right) h_{i i n}^{2}-\sum_{j=1}^{r}\left[\left(\frac{S}{\lambda_{n}^{2}}+\frac{2 S}{\lambda_{j} \lambda_{n}}+n\right) h_{n n j}^{2}+\left(\frac{S}{\lambda_{j}^{2}}+\frac{n}{3}\right) h_{j j j}^{2}\right]- \\
\sum_{j=r+1}^{t}\left[\left(\frac{S}{\lambda_{n}^{2}}+\frac{2 S}{\lambda_{j} \lambda_{n}}+n\right) h_{n n j}^{2}+\left(\frac{S}{\lambda_{g_{j}}^{2}}+\frac{2 S}{\lambda_{j} \lambda_{g_{j}}}+n\right) h_{g_{j} g_{j} j}^{2}\right]- \\
\frac{1}{3} \sum_{i \neq j \neq k}\left[\left(\frac{\lambda_{i}+\lambda_{j}+\lambda_{k}}{\lambda_{i} \lambda_{j} \lambda_{k}}\right) S+n\right] h_{i j k}^{2}-\frac{2 n}{3} S(S-n) .
\end{aligned}
$$


Notice that

$$
\left\{\begin{array}{l}
\lambda_{i} \lambda_{j}<0, i<j, \text { if and only if } i \in\{1, \ldots, n-1\} \text { and } j=n, \\
\frac{\lambda_{i}+\lambda_{j}+\lambda_{k}}{\lambda_{i} \lambda_{j} \lambda_{k}}>0, \text { for } i \neq j \neq k \in\{1, \ldots, n-1\}, \\
\frac{1}{\lambda_{g_{j}} \lambda_{j}}+\frac{1}{\lambda_{j} \lambda_{n}}>0, \text { for } j=r+1, \ldots, t, \\
n \lambda_{n}^{2}>\sum_{i} \lambda_{i}^{2}=S, \text { thus } n>\frac{S}{\lambda_{n}^{2}} .
\end{array}\right.
$$

Using Lemma 2.1 and combining (2.4) with (2.5), we get

$$
S \Delta F \leq-\frac{2 n}{3} S(S-n) .
$$

Since $M$ is compact, we can find a point $p$ on $M$ such that $F(p)=\min F$. In particular, $\nabla F(p)=0$ and $\Delta F(p) \geq 0$ at $p$. On the other hand, from $S>0, \Delta F(p) \geq 0$ and (2.6), we conclude that $S \in(0, n]$. In this situation, a theorem of (Chern et al. 1970) implies that $S=n$ so that $M$ is isometric to a Clifford Torus $S^{1}\left(\sqrt{\frac{1}{n}}\right) \times S^{n-1}\left(\sqrt{\frac{n-1}{n}}\right)$. The Corollaries 1.2 and 1.3 are obvious from the result due to (Chern et al. 1970) and Theorem 1.1.

\section{SKETCH OF THE PROOF OF THEOREM 1.4}

The proof of Theorem 1.4 follows essentially the pattern of the proof of Theorem 1.1 taking into account the presence of one term that contains $\Delta S$. We only stress those points which may lead to some differences. By using (1.10), (1.11), (2.2) and the fact that $K$ is constant, we obtain

$$
\begin{aligned}
0=S \Delta F=- & \sum_{i}\left(\frac{S}{\lambda_{i}^{2}}+\frac{n}{3}\right) h_{i i i}^{2}-\sum_{i \neq j}\left(\frac{S}{\lambda_{i}^{2}}+\frac{2 S}{\lambda_{i} \lambda_{j}}+n\right) h_{i i j}^{2}- \\
& \sum_{i \neq j \neq k}\left(\frac{S}{\lambda_{i} \lambda_{j}}+\frac{n}{3}\right) h_{i j k}^{2}-\frac{2 n}{3} S(S-n)+\frac{n \Delta S}{6} .
\end{aligned}
$$

Since $M$ is compact, we can find a point $p$ on $M$ such that $S(p)=\max S$. In particular, $\nabla S(p)=0$ and $\Delta S(p) \leq 0$ at $p$. Since $\nabla S(p)=0$, we have $\sum_{i} \lambda_{i} h_{i i j}(p)=0, j=1, \ldots, n$, therefore, defining $\beta_{i j}=h_{i i j}(p)$, then $\lambda_{i}(p)$ and $\beta_{i j}$ satisfy the conditions in Lemma 2.1.

By making use of the same proof as in Theorem 1.1, we obtain the following inequality

$$
0 \leq \frac{n \Delta S(p)}{6}-\frac{2 n}{3} S(p)(S(p)-n)
$$

Since $S>0, S(p)=\max S$ and $\Delta S(p) \leq 0$, by (3.2) we get that $S \in(0, n]$. It follows from a theorem of (Chern et al. 1970) that $M$ is isometric to a Clifford Torus $S^{1}\left(\sqrt{\frac{1}{n}}\right) \times S^{n-1}\left(\sqrt{\frac{n-1}{n}}\right)$. 
REMARK. Assume that $S$ is bounded from above or that $K$ is bounded from below. In this situation, by applying the Generalized Maximum Principle due to Yau and Omori (Yau 1975), and a result due to (Cheng 1993), we can generalize all the results in this note to complete hypersurfaces in $S^{n+1}$.

\section{ACKNOWLEDGEMENTS}

This work is part of my Doctoral Thesis at the Instituto de Matemática - UFRJ. I want to thank W. Santos for her supportive orientation and also M. P. do Carmo and H. Alencar for helpful conversations and suggestions. Research work supported by Faperj-RJ.

\section{RESUMO}

Seja $M$ uma hipersuperfície n-dimensional, mínima, fechada, imersa na esfera unitária $S^{n+1}$ dimensional. Suponha que $M$ tem curvatura escalar ou curvatura de Gauss-Kronecker constantes. Nesta nota, anunciaremos que se $M$ tem $(n-1)$ curvaturas principais com o mesmo sinal em todos os seus pontos, então $M$ é isométrica ao toro de Clifford $S^{1}\left(\sqrt{\frac{1}{n}}\right) \times S^{n-1}\left(\sqrt{\frac{n-1}{n}}\right)$.

Palavras-chave: Hipersuperfícies mínimas, curvatura escalar, curvaturas principais, toro de Clifford.

\section{REFERENCES}

CHeng QM. 1993. A Characterization of Complete Riemannian Manifolds Minimally Immersed in the Unit Sphere, Nagoya Math J, 131: 127-133.

Chern SS, do Carmo MP and Kobayashi S. 1970. Minimal Submanifolds of a Sphere with Second Fundamental Form of Constant Length. In Functional Analysis and Related Fields, edited by F. BROWDER, Berlin, Springer-Verlag, 59-75.

Peng CK and Terng CL. 1983. Minimal Hypersurfaces of Spheres with Constant Scalar Curvature, Seminar on Minimal Submanifolds, (E. Bombieri, ed.), Ann of Math Studies, Princeton University Press, Princeton, NJ, 177-198.

YAU ST. 1975. Harmonic Functions on Complete Riemannian Manifolds, Comm. Pure and Applied Math., 28: 201-228. 